\title{
Autophagy Functions to Prevent Methylglyoxal-Induced Apoptosis in HK-2 Cells
}

\author{
So-Hyun Park $\mathbb{D}^{1,2}$ Hyun-Il Choi $\mathbb{D}^{1},{ }^{1}$ Jiyun Ahn $\mathbb{D}^{1,2}$ Young-Jin Jang ${ }^{1}{ }^{1}$ Tae-Youl Ha $\mathbb{D}^{1,2}$ \\ Hyo-Deok Seo $₫{ }^{1},{ }^{1}$ Yoon-Sook Kim $\left(10,{ }^{1}\right.$ Dae-Hee Lee $\left(\mathbb{1},{ }^{3}\right.$ and Chang Hwa Jung $\mathbb{D}^{1,2}$ \\ ${ }^{1}$ Division of Food Functionality Research, Korea Food Research Institute, Wanju-gun, Jeollabuk-do 55365, Republic of Korea \\ ${ }^{2}$ Department of Food Biotechnology, Korea University of Science and Technology, Wanju-gun, Jeollabuk-do 55365, Republic of Korea \\ ${ }^{3}$ Department of Marine Food Science and Technology, Gangneung-Wonju National University, Gangneung, \\ Gangwon-do 25457, Republic of Korea
}

Correspondence should be addressed to Chang Hwa Jung; chjung@kfri.re.kr

Received 26 February 2020; Revised 14 April 2020; Accepted 25 April 2020; Published 4 June 2020

Academic Editor: Rodrigo Franco

Copyright (C) 2020 So-Hyun Park et al. This is an open access article distributed under the Creative Commons Attribution License, which permits unrestricted use, distribution, and reproduction in any medium, provided the original work is properly cited.

\begin{abstract}
Methylglyoxal (MGO), a reactive carbonyl species, causes cellular damage and is closely related to kidney disease, particularly diabetic nephropathy. Although MGO has been reported to induce autophagy and apoptosis, the relationships between the two pathways are unclear. Here, we evaluated whether autophagy may be the underlying mechanism inhibiting MGO-induced apoptosis. MGO treatment induced concentration- and time-dependent apoptosis in HK-2 cells. Moreover, MGO upregulated the autophagy markers p62 and LC3-II. Apoptosis caused by MGO was increased in ATG5-knockdown cells compared to that in wild-type cells. In contrast, autophagy activation by 5-aminoimidazole-4-carboxamide ribonucleotide resulted in reduced apoptosis, suggesting that autophagy played a role in protecting against MGO-induced cell death. To examine the mechanisms through which autophagy occurred following MGO stimulation, we investigated changes in AKT/mammalian target of rapamycin (mTOR) signaling. Autophagy induction by MGO treatment was not related to AKT/mTOR signaling; however, it did involve autophagy-related gene expression promoted by AMP-activated protein kinase-mediated transcription factors, such as forkhead box 1. Overall, our findings indicate that MGO-induced cellular damage can be mitigated by autophagy, suggesting that autophagy may be a potential therapeutic target for diseases such as diabetic nephropathy.
\end{abstract}

\section{Introduction}

Methylglyoxal (MGO), a reactive carbonyl species, is formed in the glycolytic pathway following fragmentation of glyceraldehyde-3-phosphate and dihydroxyacetone phosphate [1]. MGO is a major precursor of advanced glycation end products and can induce intracellular damage by increasing reactive oxygen species levels and mitochondrial damage, leading to apoptosis [2-4]. According to previous studies, MGO is produced rapidly under hyperglycemic conditions in diabetic patients, and accumulated MGO is then involved in several diseases, such as cognitive dysfunction, cardiovascular diseases, and bone loss [5-10]. In particular, MGO contributes to the development of diabetic microvascular complications, such as nephropathy and retinopathy [11-13]. Although a recent study revealed that MGO is related to the early progression of diabetic nephropathy, the molecular mechanisms of the cytotoxic effects of MGO in kidney cells remain unclear [14].

Autophagy is an intracellular degradation mechanism that targets damaged organelles, lipids, and aggregated proteins [15]. Importantly, autophagy has critical roles in cell survival and cell death responses to intracellular stress. Indeed, previous studies have shown that autophagy alleviates cell death in a variety of stress environments, including starvation, oxidative stress, and DNA damage [16-18]. Additionally, autophagy inducers, such as 5-aminoimidazole-4carboxamide ribonucleotide (AICAR) and rapamycin, also block various stress-induced cell death pathways, suggesting a correlation between MGO-induced autophagy and apoptosis $[19,20]$. Autophagy activation in human brain microvascular epidermal cells protects against MGO-induced cell 
damage [21]. Luteolin, a type of phytochemical flavonoid, also inhibits cell death in MGO-treated cells by blocking mammalian target of rapamycin (mTOR) signaling [22]. However, the effects of autophagy in MGO-induced apoptosis and its associated mechanisms have not been fully elucidated in kidney cells.

Accordingly, in this study, we investigated whether autophagy had protective effects against MGO-induced apoptosis and examined the mechanisms through which autophagy was caused by MGO.

\section{Materials and Methods}

2.1. Cell Culture. Human kidney (HK-2) and SV40MES13 cells were purchased from the American Type Culture Collection (Manassas, VA, USA). HK-2 cells were cultured in Roswell Park Memorial Institute 1640 medium supplemented with $10 \%$ fetal bovine serum (FBS) and $1 \%$ penicillin-streptomycin (PS). SV40MES13 cells were cultured in a 3:1 mixture of DMEM/F-12 medium supplemented with $5 \%$ FBS, $14 \mathrm{mM}$ of HEPES, and $1 \%$ PS at $37^{\circ} \mathrm{C}$ in an atmosphere containing $5 \% \mathrm{CO}_{2}$.

2.2. Cell Viability. Cells were seeded in 96-well plates at a density of $1 \times 10^{4}$ cells/well in $100 \mu \mathrm{L}$ complete medium and incubated for $24 \mathrm{~h}$. The cells were treated with various concentrations $(0-1 \mathrm{mM})$ of MGO with $100 \mu \mathrm{L}$ complete medium for 24 or $48 \mathrm{~h}$. Following treatment, $20 \mu \mathrm{L}$ MTT solution $(5 \mathrm{mg} / \mathrm{mL}$ in phosphate-buffered saline) was added to each well, and cells were incubated for an additional $2 \mathrm{~h}$. Next, the medium was removed, and $200 \mu \mathrm{L}$ dimethyl sulfoxide was added to each well. The cell viability was determined by measuring absorbance at $540 \mathrm{~nm}$ using an Infinite M200 microplate reader (Tecan US, Inc., Morrisville, NC, USA).

2.3. Terminal Deoxynucleotidyl Transferase dUTP Nick-End Labeling (TUNEL) Assay. Apoptotic cells were detected using a DeadEnd Fluorometric TUNEL System kit (cat. no. G3250; Promega, Madison, WI, USA) according to the manufacturer's instructions. Apoptotic cells were imaged using a fluorescent microscope (Olympus IX71, Japan).

2.4. Annexin $V$ and Dead Cell Assays. The apoptotic cell death ratio was determined using a Muse Annexin $\mathrm{V}$ and Dead Cell Assay Kit (cat. no. MCH100105; Merck Millipore, Billerica, MA, USA). Briefly, HK-2 cells were seeded into 6 -well plates $\left(2 \times 10^{5}\right.$ cells/well $)$. After $24 \mathrm{~h}$, cells were treated with 0.5 or $0.75 \mathrm{mM} \mathrm{MGO}$ for $24 \mathrm{~h}$. After treatment, all cells were harvested and stained with annexin $\mathrm{V}$ solution for 20 min. Apoptotic cell death was measured using a MUSE cell analyzer (Merck Millipore).

2.5. Immunoblotting. Cellular proteins were harvested using cell lysis buffer (cat. no. 9803S; Cell Signaling Technology, Danvers, MA, USA) supplemented with protease and phosphatase inhibitor cocktails (Thermo Fisher Scientific, Waltham, MA, USA). The harvested cells were centrifuged at $13,000 \mathrm{rpm}$ for $10 \mathrm{~min}$ at $4^{\circ} \mathrm{C}$, and the supernatants were separated. The proteins were quantified using Bradford assays and separated by sodium dodecyl sulfate polyacryl-
Table 1: Primer sequences.

\begin{tabular}{|c|c|c|}
\hline DNA target & & Sequence $\left(5^{\prime}\right.$ to $\left.3^{\prime}\right)$ \\
\hline \multirow{2}{*}{$M A P 1 L C 3 B$} & $\mathrm{~F}$ & GATGTCCGACTTATTCGAGAGC \\
\hline & $\mathrm{R}$ & TTGAGCTGTAAGCGCCTTCTA \\
\hline \multirow{2}{*}{ BECN11 } & $\mathrm{F}$ & CCATGCAGGTGAGCTTCGT \\
\hline & $\mathrm{R}$ & GAATCTGCGAGAGACACCATC \\
\hline \multirow{2}{*}{ FOXO3 } & $\mathrm{F}$ & CGGACAAACGGCTCACTCT \\
\hline & $\mathrm{R}$ & GGACCCGCATGAATCGACTAT \\
\hline \multirow{2}{*}{ TFE3 } & $\mathrm{F}$ & CCGTGTTCGTGCTGTTGGA \\
\hline & $\mathrm{R}$ & GCTCGTAGAAGCTGTCAGGAT \\
\hline \multirow{2}{*}{ TFEB } & $\mathrm{F}$ & ACCTGTCCGAGACCTATGGG \\
\hline & $\mathrm{R}$ & CGTCCAGACGCATAATGTTGTC \\
\hline \multirow{2}{*}{ FOXO1 } & $\mathrm{F}$ & TCGTCATAATCTGTCCCTACACA \\
\hline & $\mathrm{R}$ & CGGCTTCGGCTCTTAGCAAA \\
\hline \multirow{2}{*}{ CREB } & $\mathrm{F}$ & TCAGCCGGGTACTACCATTC \\
\hline & $\mathrm{R}$ & TCTCTTGCTGCTTCCCTGTT \\
\hline \multirow{2}{*}{$G A B A R A B$} & $\mathrm{~F}$ & AGAAGAGCATCCGTTCGAGAA \\
\hline & $\mathrm{R}$ & CCAGGTCTCCTATCCGAGCTT \\
\hline \multirow{2}{*}{ ATG16L } & $\mathrm{F}$ & AACGCTGTGCAGTTCAGTCC \\
\hline & $\mathrm{R}$ & AGCTGCTAAGAGGTAAGATCCA \\
\hline \multirow{2}{*}{$B N I P 3$} & $\mathrm{~F}$ & CAGGGCTCCTGGGTAGAACT \\
\hline & $\mathrm{R}$ & CTACTCCGTCCAGACTCATGC \\
\hline \multirow{2}{*}{ ACTIN } & $\mathrm{F}$ & CATGTACGTTGCTATCCAGGC \\
\hline & $\mathrm{R}$ & CTCCTTAATGTCACGCACGAT \\
\hline \multirow{2}{*}{$A M P K \alpha$} & $\mathrm{F}$ & TTG AAA CCT GAA AAT GTC CTG CT \\
\hline & $\mathrm{R}$ & GGT GAG CCA CAA CTT GTT CTT \\
\hline \multirow{2}{*}{ ATG5 } & $\mathrm{F}$ & TGTGCTTCGAGATGTGTGGTT \\
\hline & $\mathrm{R}$ & GTCAAATAGCTGACTCTTGGCAA \\
\hline \multirow{2}{*}{ ATG12 } & $\mathrm{F}$ & TAGAGCAACACGAACCATCC \\
\hline & $\mathrm{R}$ & CACTGCCAAAACACTCATAGAGA \\
\hline \multirow{2}{*}{ p62/SQSTM-1 } & $\mathrm{F}$ & GACTACGACTTGTGTAGCCTC \\
\hline & $\mathrm{R}$ & AGTGTCCGTGTTTCACCTTCC \\
\hline
\end{tabular}

amide gel electrophoresis. The separated proteins were transferred to polyvinylidene fluoride membranes. The membranes were then blocked with $5 \%$ skim milk for $1 \mathrm{~h}$ and incubated overnight at $4{ }^{\circ} \mathrm{C}$ with the following primary antibodies: anti-phospho-AMP-activated protein kinase (AMPK; Thr172; cat. no. 2535; Cell Signaling Technology), anti-AMPK (cat. no. 2793; Cell Signaling Technology), anti-microtubule-associated protein $1 \mathrm{~A} / 1 \mathrm{~B}$ light chain $3 \mathrm{~B}$ (LC3B; cat. no. 2775; Cell Signaling Technology), antiphospho-ribosomal protein S6 kinase $\beta-1$ (S6K1; Thr389; cat. no. 9234; Cell Signaling Technology), anti-S6K1 (cat. no. 9202; Cell Signaling Technology), anti-poly-ADP ribose polymerase (PARP; cat. no. 9542; Cell Signaling Technology), anti-p62/SQSTM-1 (cat. no. P0067; Sigma-Aldrich, St. Louis, MO, USA), anti-Atg5 (cat. no. NB110-53818; Novus 


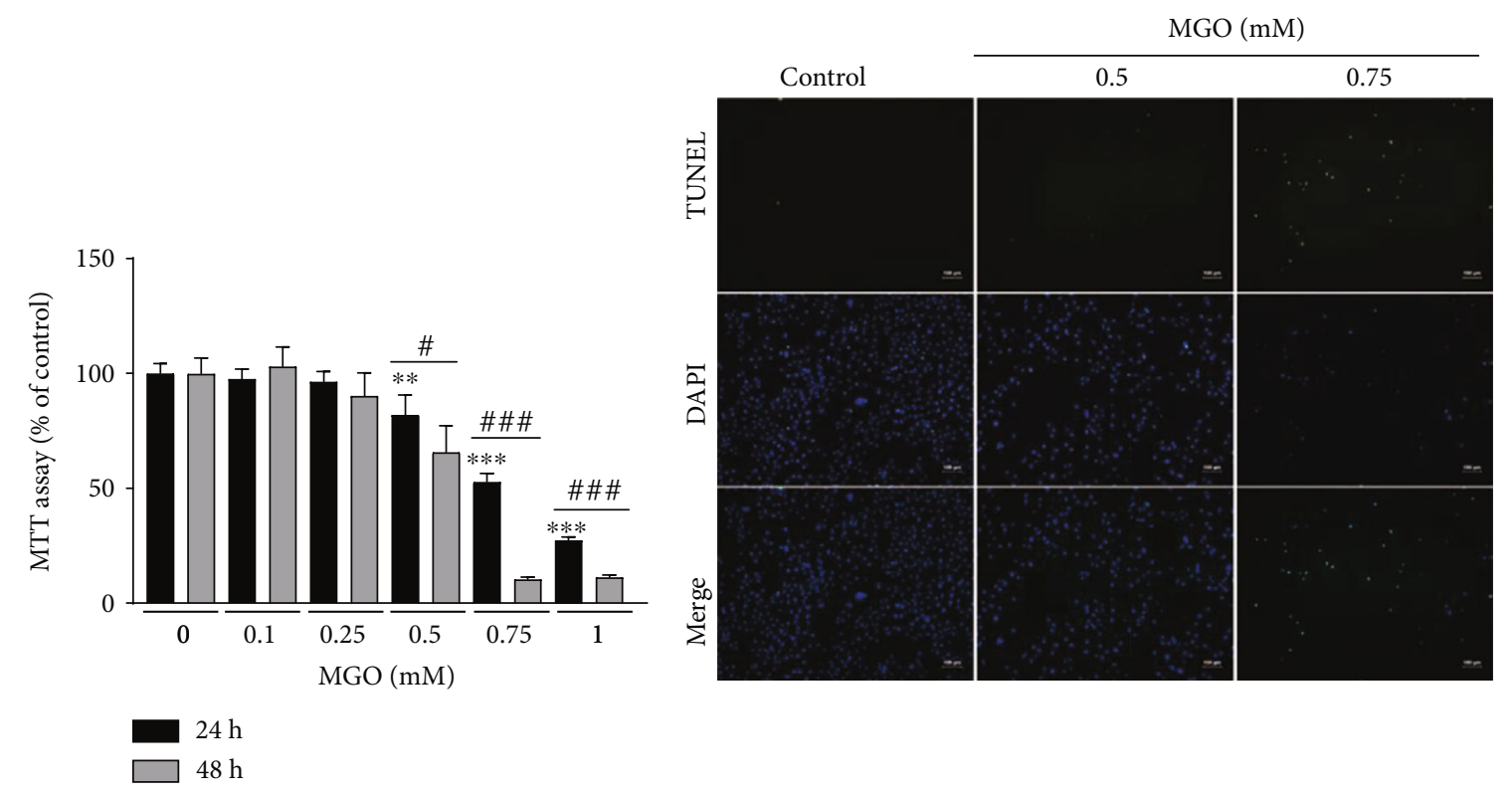

(a)

$\operatorname{MGO}(\mathrm{mM})$ :
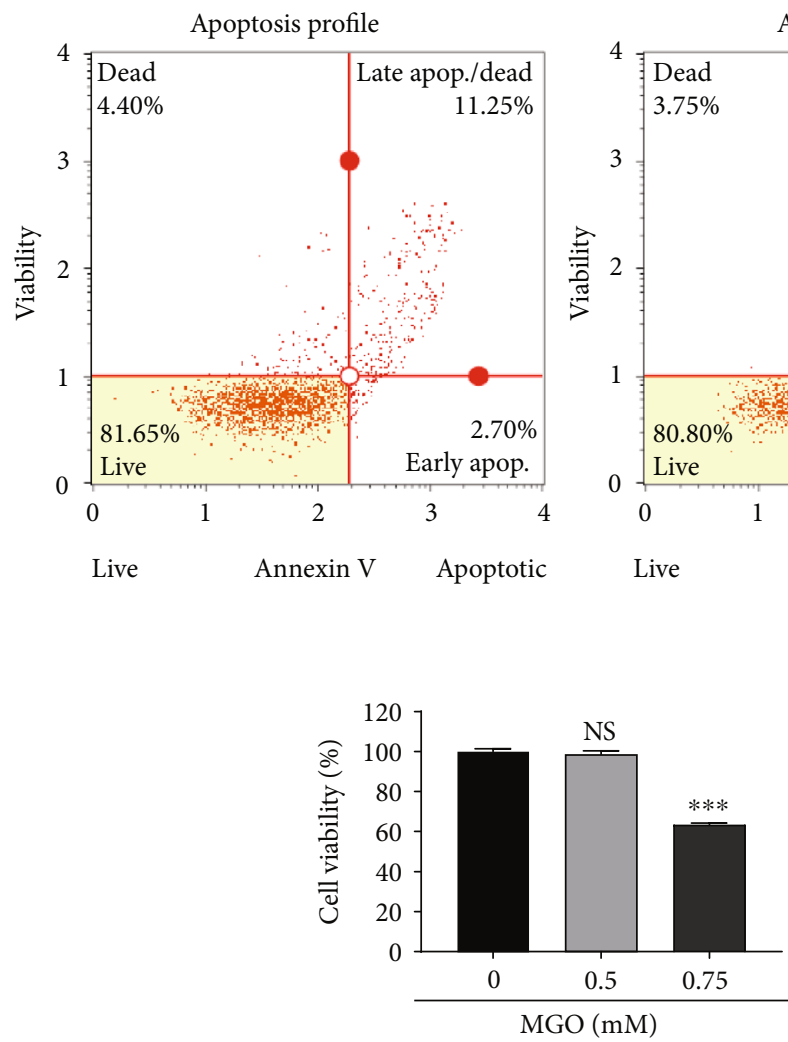

(d)

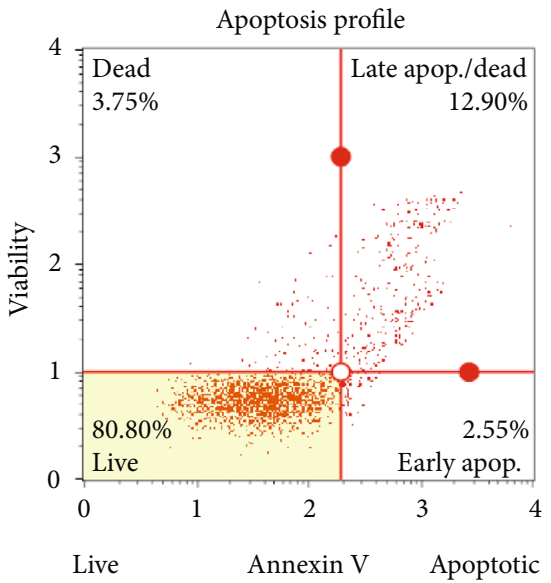

(c)

(b)

0.5

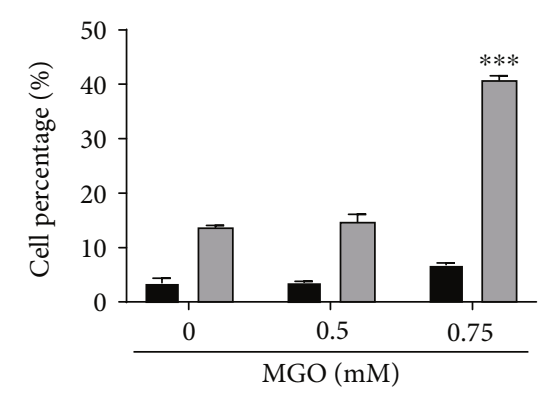

Necrotic

Apoptotic

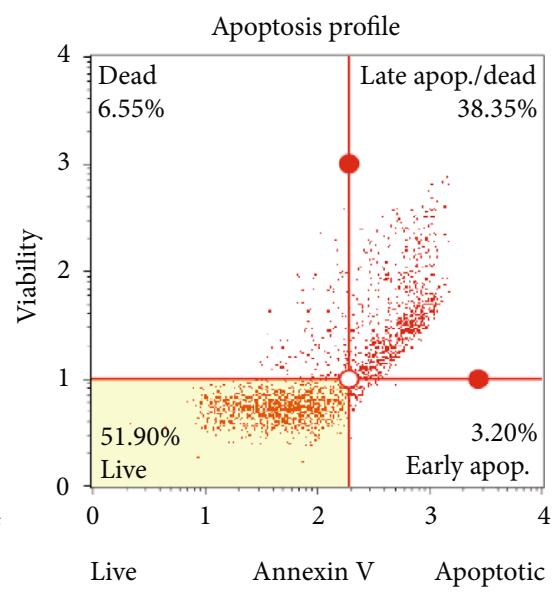

(e)

FIGURE 1: MGO treatment induces cell death in HK-2 cells. (a) HK-2 cells were treated with the indicated concentrations (0-1 mM) of MGO for 24 or $48 \mathrm{~h}$, and MTT assays were conducted. (b) Representative image of apoptotic cells in HK-2 cells treated with MGO for $24 \mathrm{~h}$ detected by TUNEL assays (original magnification: 100x). (c-e) Representative flow cytometry analysis of HK-2 cells treated with MGO for $24 \mathrm{~h}$ and then stained with the Muse Annexin V and Dead Cell Kit. (c) Flow cytometry density plot of MGO-treated HK-2 cells. (d) Cell viability and (e) total apoptotic cell percentages following treatment with $0-0.75 \mathrm{mM} \mathrm{MGO}$. Values are mean \pm standard deviation. ${ }^{* *} p<0.01$ and ${ }^{* * *} p<0.001$ versus the control ( 0 ) group; ${ }^{\#} p<0.05$ and ${ }^{\# \# \#} p<0.001$ versus the same concentration of MGO $(n=3)$. 


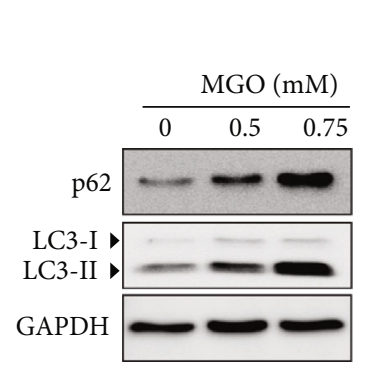

(a)

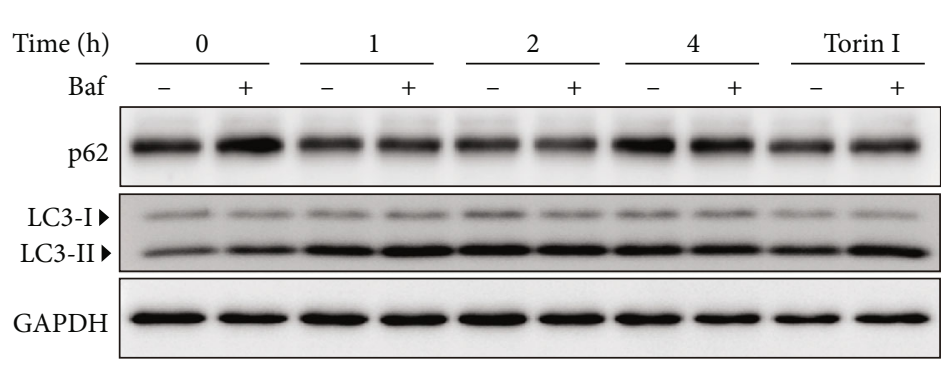

(b)

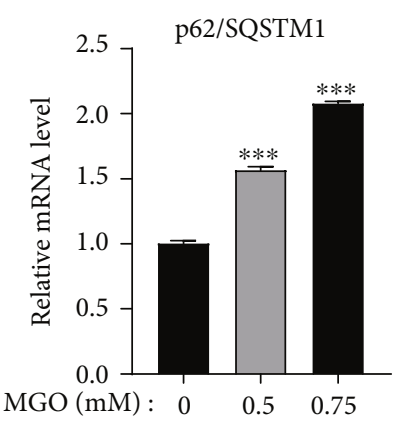

(c)

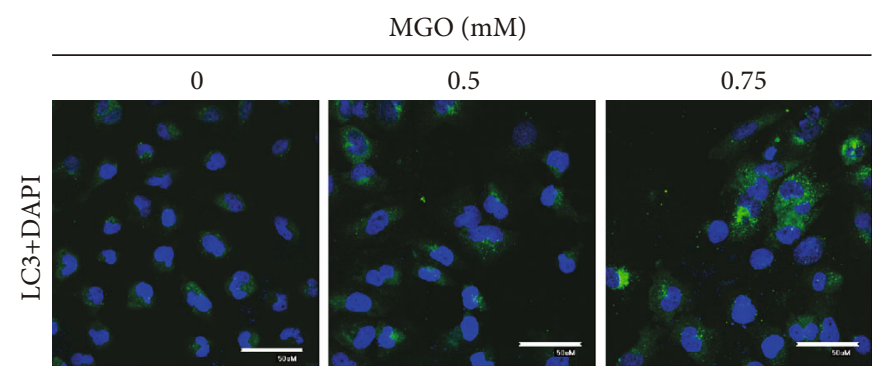

(d)

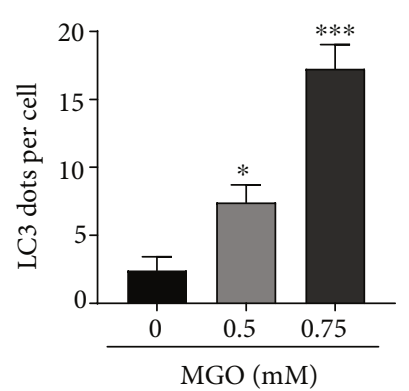

(e)

FIGURE 2: MGO treatment enhances autophagy. (a) Expression of autophagy-related proteins in HK-2 cells treated with MGO (0.5-0.75 mM) for $24 \mathrm{~h}$. (b) The time-dependent protein expression levels of LC3 and p62 in HK-2 cells treated with MGO (0.75 mM) and torin 1 (positive control, $200 \mathrm{nM}, 4 \mathrm{~h}$ ) with or without Baf $(50 \mathrm{nM}, 1 \mathrm{~h})$. (c) Transcriptional levels of p62/SQSTM-1 mRNA. (d) Representative immunofluorescence image of HK-2 cells treated with MGO for $24 \mathrm{~h}$ (green: LC3; blue: DAPI; original magnification: 600x). (e) LC3 puncta following treatment with MGO. Values are mean \pm standard deviation. ${ }^{*} p<0.05$ and ${ }^{* * *} p<0.001$ versus vehicle $(0)$ group.

Biologicals, CO, USA), anti-Caspase-3 (cat. no. 9662; Cell Signaling Technology), anti-glyceraldehyde 3-phosphate dehydrogenase (cat. no. sc-365062; Santa Cruz Biotechnology), anti-Cytochrome C (cat. no. sc-13156, Santa Cruz Biotechnology), and anti- $\beta$-actin (cat. no. sc-47778; Santa Cruz Biotechnology). The next day, the membranes were washed and incubated with secondary antibodies for $1 \mathrm{~h}$ at room temperature. Protein bands were visualized using G:BOX Chemi XX6 (Syngene Ltd., Frederick, MD, USA) with an enhanced chemiluminescence substrate. The intensities of the protein bands were measured using ImageJ software ver. 1.8 (National Institutes of Health, MD, USA).

2.6. Real-Time Quantitative Polymerase Chain Reaction (RT$q P C R)$. RNA extraction and cDNA synthesis were performed using a RNeasy Mini Kit (Qiagen, Inc., Valencia, CA, USA) and a ReverTra Ace qPCR RT Kit (Toyobo Co., Ltd., Osaka, Japan) according to the manufacturer's instructions. qPCR was then performed using the SYBR Green Realtime PCR Master Mix (Toyobo Co., Ltd.) with a Viia 7 Real-Time PCR system (Thermo Fisher Scientific). Relative RNA levels were normalized according to the levels of actin mRNA. The specific primer sequences are listed in Table 1.

2.7. Immunofluorescence. HK-2 cells were seeded into 8-well chamber slides $\left(2.5 \times 10^{4}\right.$ cells/well $)$. After $24 \mathrm{~h}$, cells were treated with MGO. Immunostaining was performed as previously described [23]. Briefly, cells were fixed with $4 \%$ formal- dehyde for $15 \mathrm{~min}$ and permeabilized with $0.05 \%$ saponin for $30 \mathrm{~min}$ at room temperature. Cells were incubated with $1 \%$ bovine serum albumin blocking solution and reacted with anti-LC3B antibody (cat. no. 2775; Cell Signaling Technology) overnight at $4^{\circ} \mathrm{C}$. The next day, cells were washed thrice and stained with rabbit secondary antibody (cat. no. 4412; Cell Signaling Technology). After $30 \mathrm{~min}$, the cells were washed with PBS and incubated with DAPI solution (cat. no. 10236276001; Sigma-Aldrich) for $5 \mathrm{~min}$. Microscopy was performed at $408 \mathrm{~nm}$ (4'6-diamidino-2-phenylindole) and $488 \mathrm{~nm}$ (fluorescein isothiocyanate) using an Eclipse Ti confocal fluorescent microscope (Nikon, Tokyo, Japan).

2.8. siRNA Transfection. siRNAs targeting Atg5 and AMPK $\alpha$ and nontargeted siRNA (siNC) were purchased from Dharmacon (CO, USA). HK-2 cells were seeded into 6-well plates $\left(2 \times 10^{5}\right.$ cells/well $)$ and incubated until at least $60 \%$ confluence was attained. The cells were transfected with siAtg5 or siAMPK or siNC using Lipofectamine RNAi Max (Thermo Fisher Scientific) according to the manufacturer's instructions. siRNA was added to Opti-MEM with a transfection reagent and incubated at room temperature for $20 \mathrm{~min}$. The mixture was then added to each well, and the cells were incubated for an additional $24 \mathrm{~h}$. After transfection, cells were harvested and used for subsequent experiments.

2.9. Statistical Analysis. All data are presented as means \pm standard deviations. The significance of differences was 

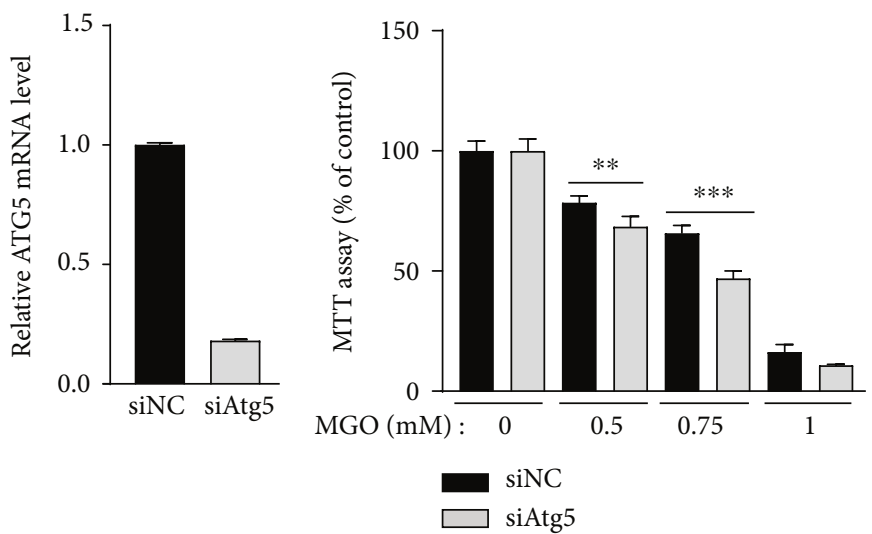

(a)

(b)
$\operatorname{MGO}(\mathrm{mM})$
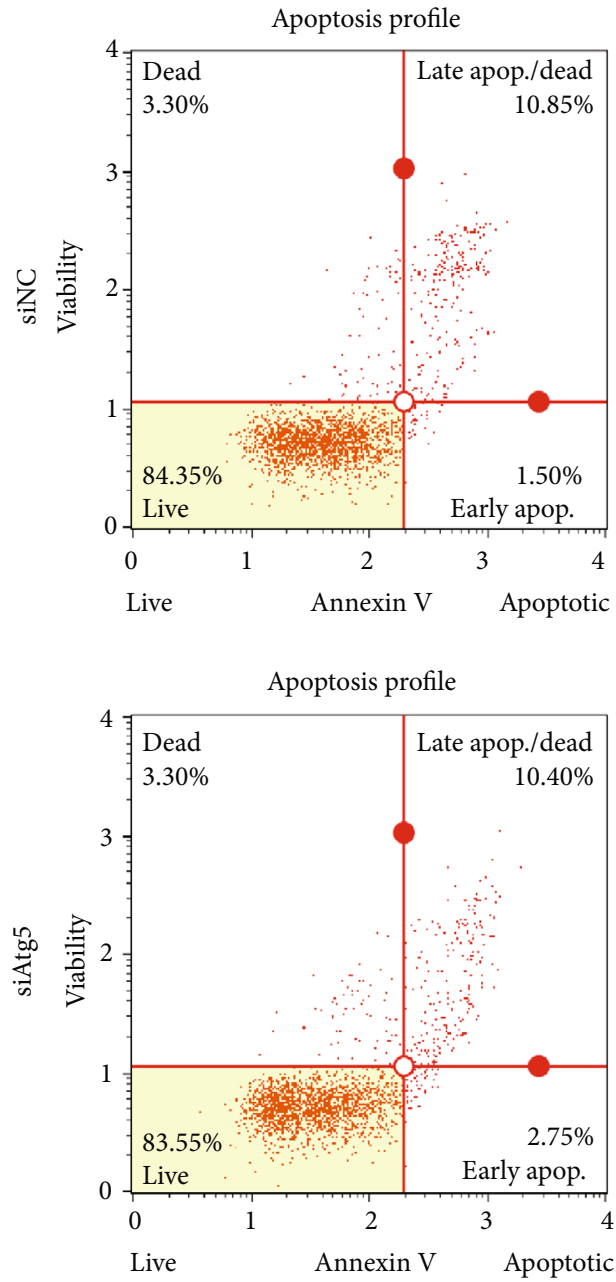

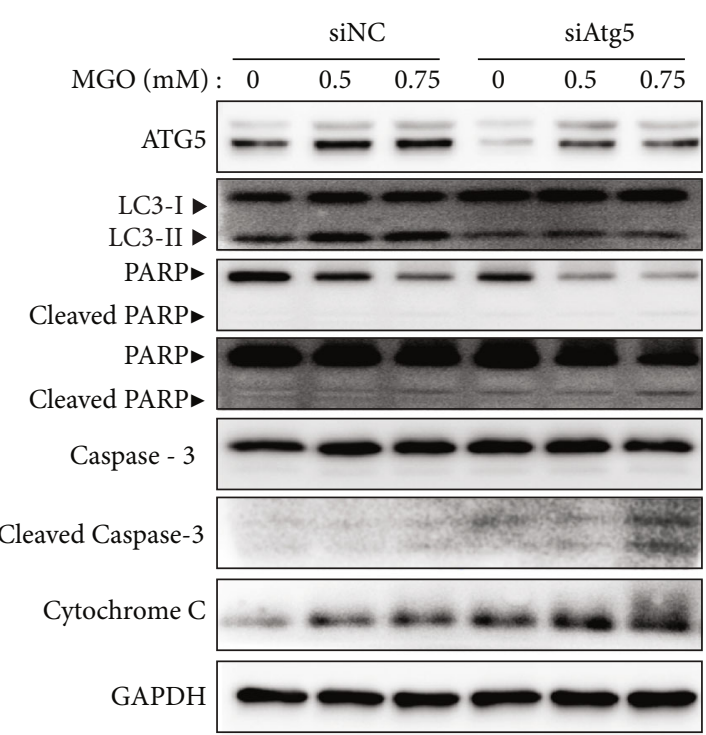

(c)

0.75

Apoptosis profile

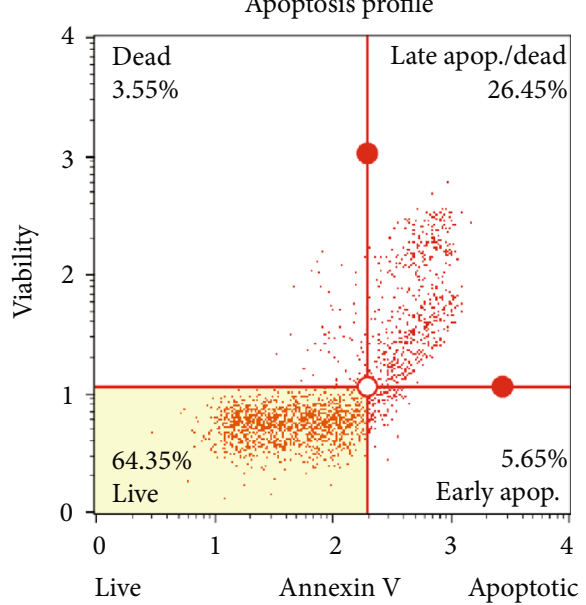

Apoptosis profile

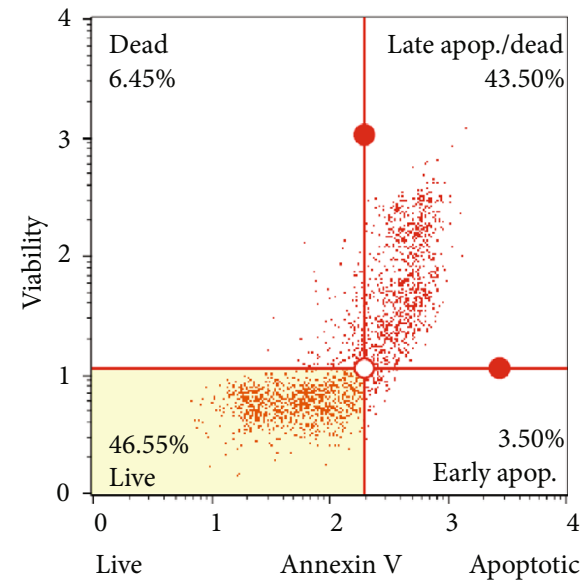

(d)

Figure 3: Continued. 


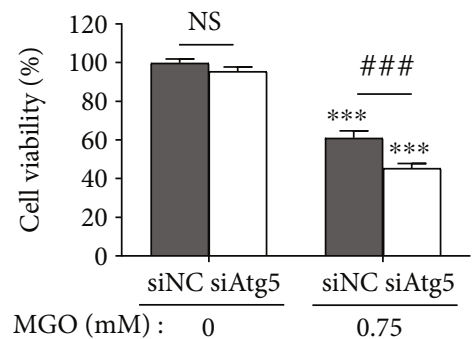

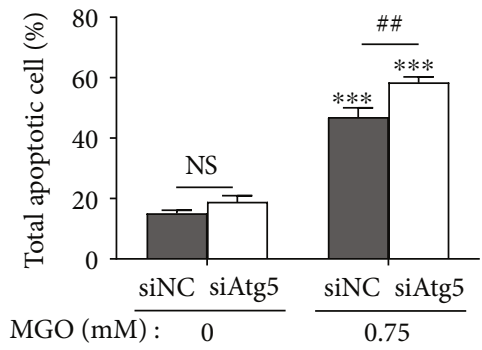

(e)
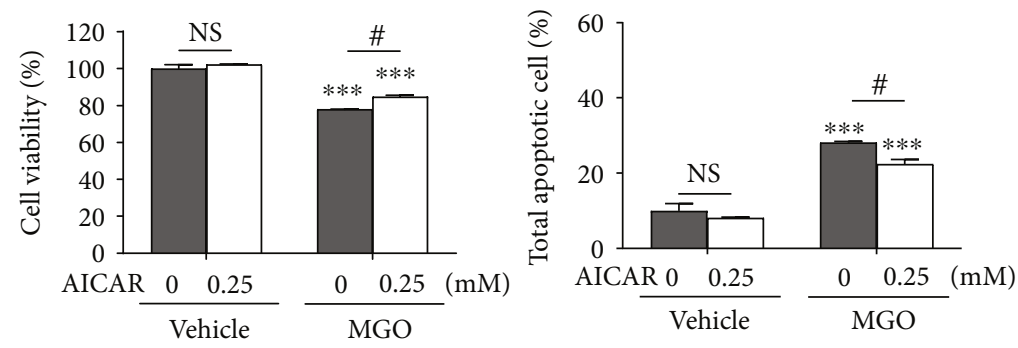

(f)

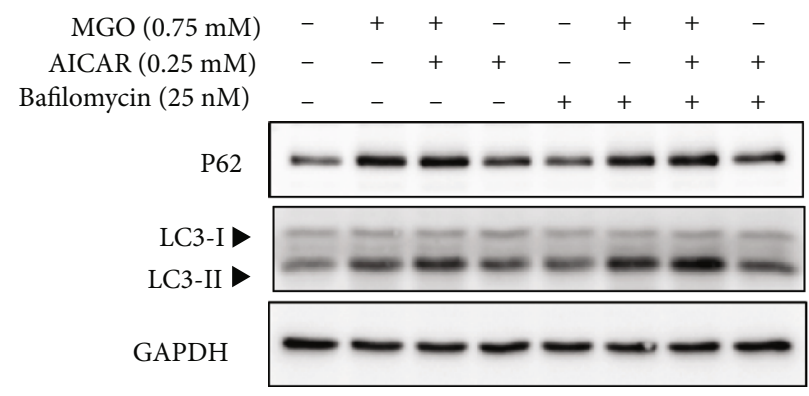

(g)

Figure 3: Autophagy exerts protective effects against MGO-induced apoptosis. (a) Relative Atg5 mRNA levels of siNC and siAtg5 HK-2 cells. (b) Viability of transient siAtg5 cells treated with MGO (0-1 mM), as determined by MTT assays. (c) Comparison of apoptosisand autophagy-related proteins in control cells and transient siAtg5-transfected cells treated with $0-0.75 \mathrm{mM}$ of MGO for $24 \mathrm{~h}$. (d) Representative images of flow cytometric analysis of apoptotic populations in control cells and transient siAtg5-transfected cells treated with $0.75 \mathrm{mM}$ MGO for $24 \mathrm{~h}$. (e) Percentages of live and apoptotic cells in the control and transient siAtg5-transfected groups treated with MGO. (f) Percentages of live and apoptotic cells cotreated with $0.75 \mathrm{mM}$ MGO for $24 \mathrm{~h}$ and $0.25 \mathrm{mM}$ AICAR for $8 \mathrm{~h}$, as determined by flow cytometric analysis. (g) Expression of autophagy-related proteins in cells treated with MGO ( $24 \mathrm{~h})$, AICAR ( $8 \mathrm{~h})$, and baf $(2 \mathrm{~h})$. Values are mean \pm standard deviation. ${ }^{* *} p<0.01$ and ${ }^{* * *} p<0.001$ versus the control (0) group; ${ }^{\#} p<0.05,{ }^{\# \#} p<0.01$, and ${ }^{\# \# \#} p<0.001$ versus the same concentration of MGO ( $n=3$ experiments).

evaluated by one-way analysis of variance, followed by Tukey's post hoc multiple comparison test using GraphPad Prism 7 software (GraphPad Software, San Diego, CA, USA). Differences with $p$ values of less than 0.05 were considered significant.

\section{Results}

3.1. MGO Treatment Induced Apoptosis in HK-2 Cells. MGO is known to induce cell death and suppress cell proliferation $[24,25]$. Therefore, we first evaluated the appropriate concentration of MGO for reducing cell viability in HK-2 cells using MTT assays with various concentrations of MGO. The results showed that MGO decreased cell viability in a concentrationand time-dependent manner and significantly induced cell death following treatment with MGO for $24 \mathrm{~h}$ at a concentration above $0.5 \mathrm{mM}$ (Figure 1(a), Figure $\mathrm{S} 1(\mathrm{a})$ ). We then confirmed the apoptosis-inducing concentration $(0.75 \mathrm{mM})$ using TUNEL assays, showing increased formation of green dots representing DNA fragmentation (Figure 1(b)). Muse Annexin V and Dead Cell Assays showed that the proportion of apoptotic cells was significantly increased following treatment with $0.75 \mathrm{mM}$ MGO; however, the increase in necrotic cell death was not significant (Figures 1(c)-1(e)). These results indicate that treatment with $0.75 \mathrm{mM}$ MGO for $24 \mathrm{~h}$ in $\mathrm{HK}-2$ cells induced apoptosis in approximately $40 \%$ of the cells.

3.2. MGO Treatment Induced Autophagy. According to recent reports, MGO increases autophagy in cells $[25,26]$. Therefore, we next investigated whether autophagy occurs at apoptosis-inducing MGO concentrations. Autophagy protein markers, such as p62/SQSTM-1 and LC3-II, were increased in a concentration-dependent manner following 


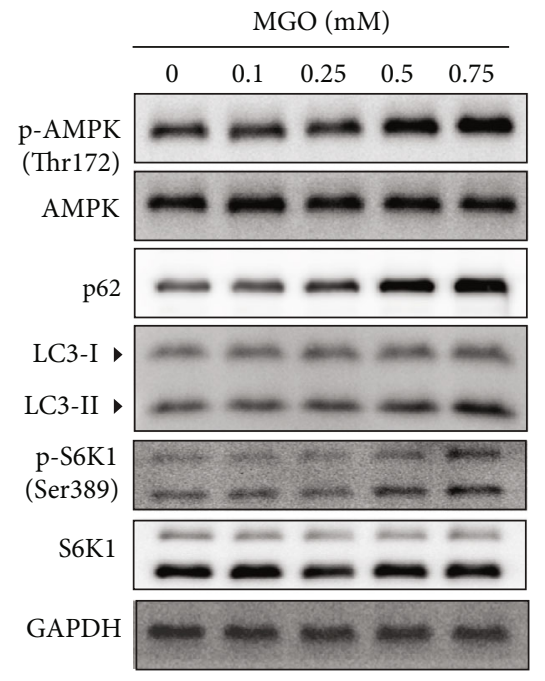

(a)

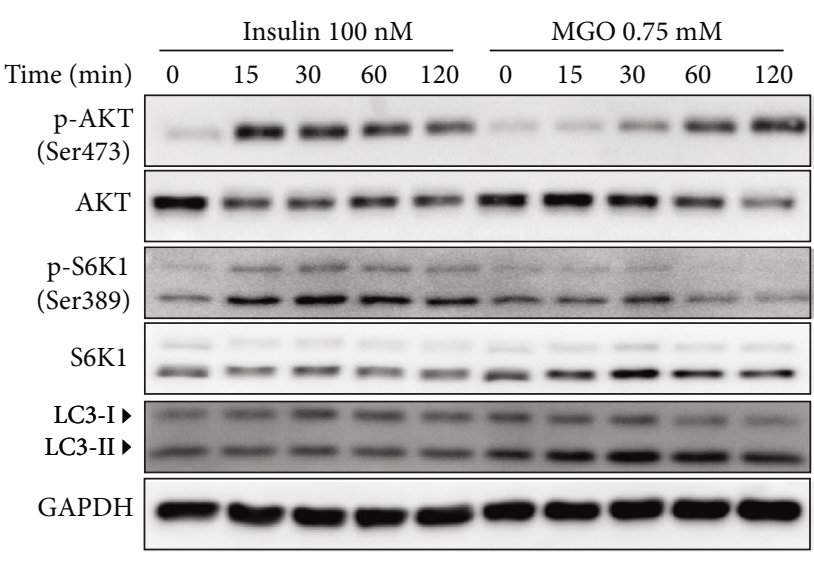

(b)

FIGURE 4: MGO upregulates autophagy independently of the mTORC pathway. (a) Western blot analysis of the effects of MGO treatment for $24 \mathrm{~h}$ on AMPK and autophagy-related proteins. (b) Time-dependent protein expression of MGO (0-120 min) or insulin treatment in serumfree media conditions $(16 \mathrm{~h})$.

treatment with MGO (Figure 2(a)). In general, when autophagy is activated, LC3-II protein expression is increased while the expression of p62/SQSTM-1 is decreased [27]. Alternatively, the inhibition of autophagy flux can induce the accumulation of p62/SQSTM-1. We, therefore, sought to evaluate autophagy flux using bafilomycin A1 (baf), an autophagosome-lysosome fusion inhibitor, and found that MGO significantly increased protein levels of LC3 over $1-4 \mathrm{~h}$, and the accumulation of LC3 induced by baf was increased in MGO-treated cells compared with that in baf control cells, similarly to what was observed for torin 1 (autophagy activator, positive control) (Figure 2(b)). In addition, our data showed that MGO enhanced p62/SQSTM-1 mRNA (Figure 2(c)), suggesting that MGO activated autophagy flux, and the autophagy enhancement was associated with p62/SQSTM-1 mRNA expression. Moreover, the numbers of autophagosome puncta in MGO-treated cells was increased compared with that in the control group (Figures 2(d) and 2(e)). Collectively, our results suggested that MGO enhanced autophagy in HK-2 cells and increased the expression of autophagy-related gene.

\subsection{Autophagy Had Protective Effects against MGO-Induced} Apoptosis. Autophagy can induce autophagic cell death [28]. Therefore, we verified whether MGO-activated autophagy induces cell death or protects damaged cells. To confirm the role of autophagy in MGO-treated cells, we transfected them with Atg5 siRNA, a key subunit involved in autophagosome formation, and measured cell viability using MTT assays [29]. The viability of MGO-treated siAtg5 cells was found to be significantly lower than that of control cells (Figures 3(a) and 3(b)). Moreover, MGO increased the levels of apoptosis marker proteins, such as PARP, caspase3 , and cytochrome C, in siATG5 cells compared with their levels in siNC cells, suggesting that autophagy may protect cells from MGO-induced cell death (Figure 3(c)). Interest- ingly, MGO also increased Atg5 protein expression in both siAtg 5 and siNC cells, suggesting that MGO contributes to the upregulation of autophagy-related proteins, such as p62 and Atg5. We next analyzed the percentage of apoptotic cells in siAtg5 cells treated with MGO and found that cell viability was decreased by approximately $15 \%$ compared with that of siNC cells. Additionally, the percentage of apoptotic cells among siAtg5 cells treated with MGO was approximately $12 \%$ higher than that among siNC cells (Figures $3(\mathrm{~d})$ and $3(\mathrm{e}))$. We then investigated whether autophagy-activating drugs can reduce MGO-induced apoptosis. To this end, we treated cells with AICAR, an autophagy activator that acts via AMPK activation, along with MGO and found that AICAR significantly suppressed MGO-induced apoptosis (Figure 3(f)). Treatment with AICAR has been shown to further increase autophagy flux, and this suggests that increasing autophagy activity may reduce MGO-induced cell death (Figure $3(\mathrm{~g})$ ). Taken together, these results suggest that MGO-induced autophagy does not contribute to apoptosis, but rather protects against cell death.

3.4. MGO-Induced Autophagy Occurred via AMPK Independent of the mTOR Complex 1 (mTORC1) Pathway. MGO has been reported to act as an AMPK activator [26]. In this study, we confirmed that AMPK phosphorylation was increased in a concentration-dependent manner following MGO treatment (Figure 4(a), Figure S2). Interestingly, MGO also increased the expression of p-S6K1, an mTOR signaling protein. Since the mTOR pathway negatively regulates autophagy [30], it is necessary to confirm the effect of MGO on mTOR signaling. To this end, we treated HK-2 cells with insulin (mTOR signaling inducer) and MGO in serum-free conditions. Insulin treatment stimulated the phosphorylation of AKT at Ser473 and S6K1 at Ser389, which indicates the activation of $\mathrm{mTORC} 1$ signaling without altering the LC3 protein level. Alternatively, MGO treatment 


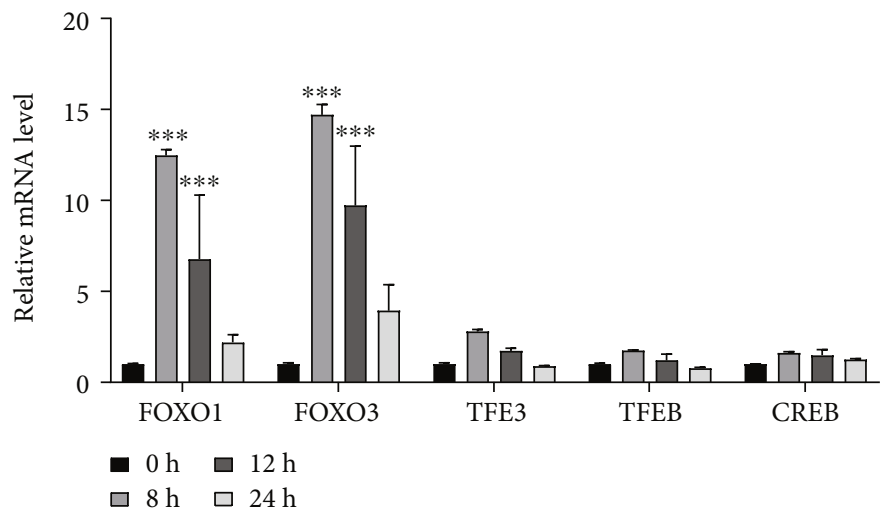

(a)
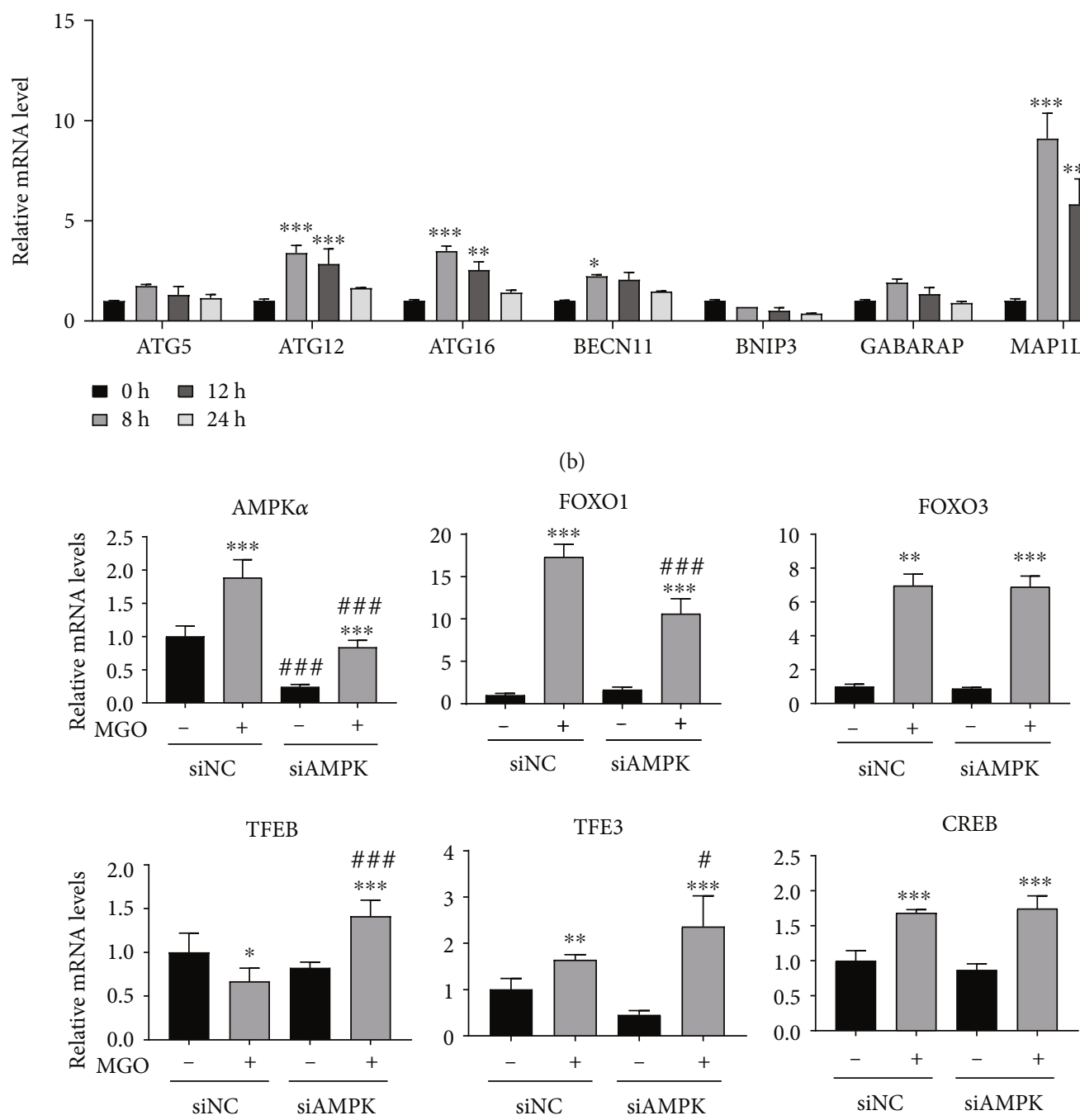

(c)

FIgURe 5: Continued. 

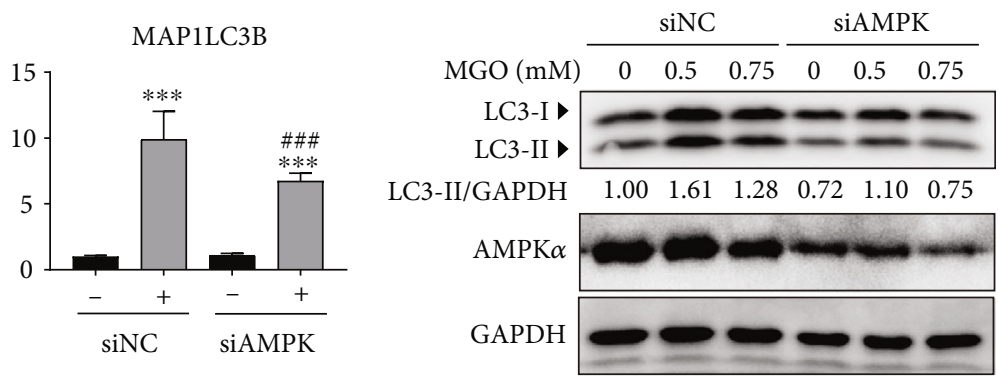

(d)

FIGURE 5: MGO upregulated the expression of autophagy-related transcription factors by enhancing AMPK activity. (a) Time-dependent mRNA levels of autophagy-related transcription factors induced by $0.75 \mathrm{mM}$ of MGO. (b) mRNA levels of autophagy subunits modulated by transcription factors in HK-2 cells treated with MGO $(0.75 \mathrm{mM})$. (c) Transcription factor mRNA levels of transient siAMPKtransfected cells compared with those of control cells treated with MGO for $8 \mathrm{~h}$. (d) Expression of MAP1LC3B mRNA and LC3 protein level in siNC or siAMPK cells following treatment with MGO for $8 \mathrm{~h}(0.75 \mathrm{mM})$ or $24 \mathrm{~h}(0-0.75 \mathrm{mM})$, respectively. Values are mean \pm standard deviation. ${ }^{*} p<0.05,{ }^{* *} p<0.01$, and ${ }^{* * *} p<0.001$ versus the control (0) group; ${ }^{*} p<0.05$ and ${ }^{\# \#} p<0.001$ versus the same concentration of MGO ( $n=3$ experiments).

not only activated the phosphorylation of AKT and S6K1, but also increased LC3-II expression (Figure 4(b)). Taken together, these results suggest that MGO activated both autophagy and mTOR signaling and that autophagy is activated through an mTOR-independent pathway.

\subsection{MGO Increased the Expression of Autophagy-Related} Transcription Factors by Enhancing AMPK Activity. Another pathway for increasing autophagy may be transcription factor regulation. Activated AMPK regulates the forkhead box (FOXO) family of transcription factors [31]. Therefore, we analyzed mRNA levels of autophagy-related transcription factors, such as FOXO1, FOXO3, transcription factor EB (TFEB), transcription factor II E (TFE), and cAMP response element-binding protein [32]. FOXO1 and FOXO3 were significantly increased by MGO treatment (Figure 5(a)). Next, autophagy-related genes, such as Atg12, BNIP3, Beclin1, GABARAP, Atg16, and Atg7, which are transcriptionally regulated by FOXOs, were analyzed. MGO increased the expression of most autophagy genes, except BNIP3 (Figure 5(b)). To verify the influence of activated AMPK on transcription factor expression, we used transient siAMPKtransfected cells to confirm enhancement of transcription factor expression by AMPK activation. The results showed that FOXO1 expression, but not FOXO3 expression, was decreased by MGO treatment in siAMPK-transfected cells (Figure 5(c)). In addition, the expression of LC3B, which is regulated by FOXO1, was also significantly reduced by AMPK silencing (Figure 5(d)). These results indicated that MGO upregulated the mRNA expression of FOXO1 autophagy-related transcription factors by enhancing AMPK activity.

\section{Discussion}

Autophagy has been shown to inhibit or induce apoptosis in different stress environments; therefore, understanding the effects of autophagy and its mechanisms in different situations remains a major research focus [33]. In the absence of autophagy, MGO-induced apoptosis was signifi- cantly increased and treatment with an autophagy activator in addition to MGO significantly increased cell survival. Thus, autophagy in dicarbonyl stress appears to have a protective role in cells.

Although autophagy is controlled through various mechanisms, it is known that the mTOR pathway is a major regulatory pathway in autophagy [30]. Likewise, drugs known as mTOR inhibitors, namely, rapamycin and torin 1 , have been reported to significantly increase autophagy [34, 35]. Moreover, AMPK is a master regulator of cellular energy homeostasis and has been shown to induce autophagy via inhibiting mTORC1 signaling through phosphorylation of raptor and TSC2, while also activating ULK1, which is associated with autophagy initiation [36]. Interestingly, our study demonstrated that MGO treatment activates S6K1, a protein downstream of mTORC1. Furthermore, in serumfree conditions, MGO not only induced autophagy but also increased AKT and S6K1 activity, similar to that observed following treatment with insulin. Normally, nutrient deficiency increases autophagy and suppresses the mTOR pathway. These results demonstrate that MGO increases autophagy and mTOR signaling, even under starvation conditions. Furthermore, MGO-induced autophagy was regulated via pathways independent of mTOR. However, both the induction of autophagy and mTOR signaling require further investigation.

AMPK also upregulates autophagy through the activation of specific transcription factors, including FOXOs, TFEB, TFE3, and CREB, which effectively upregulate autophagy by increasing the expression of autophagy-related genes [3739]. Specifically, activated AMPK phosphorylates the Ser 383 and Thr649 of FOXO1 to serve as a nuclear transcription factor. Alternatively, AKT phosphorylates the Thr24 and Ser256 of FOXO1, promoting the degradation of cytosolic FOXOs. Since MGO activated both AMPK and AKT, it is difficult to determine the effect of MGO on the transcriptional activity of FOXO1. However, according to a previous study, AMPKmediated Ser22 phosphorylation of FOXO1 inhibits Thr24 phosphorylation by AKT, and subsequently promotes the degradation of FOXO1 [40]. Our data indicate that the 
activity of FOXO1 is directly regulated by AMPK in MGOtreated cells. Therefore, FOXO1 may be regulated by AMPK rather than AKT in MGO-treated HK-2 cells.

Specific limitations were noted in this study. First, all conclusions were drawn from in vitro analyses alone. Although, recently, diabetic nephropathy preclinical animal models have been reported, most of these mouse models only partially reflect the phenotype of diabetic nephropathy, such as hyperfiltration, mesangial expansion, and glomerular basement membrane thickening. Therefore, novel nephropathy models of complete diabetic kidney disease are needed to validate our findings [41]. Second, further studies are needed to determine whether increasing the activity of autophagy in diabetic nephropathy is effective in alleviating diabetic complications.

\section{Conclusions}

The data obtained through this study indicate that MGO induces both apoptosis and autophagy. MGO induced autophagy by inducing AMPK activity independently of mTOR signaling. Further, we found that autophagy reduced $\mathrm{MGO}$-induced apoptosis through AMPK activation. MGO-activated AMPK increased FOXO1 expression, an autophagy-related transcription factor, resulting in an increased expression of autophagy-related genes. These findings are expected to be useful in carbonyl stressrelated diseases, such as diabetic nephropathy treatment and prevention strategies.

\section{Data Availability}

The data used to support the findings of this study are available from the corresponding authors upon request.

\section{Conflicts of Interest}

The authors declare that there is no conflict of interest regarding the publication of this paper.

\section{Authors' Contributions}

So-Hyun Park and Hyun-Il Choi contributed equally to this work.

\section{Acknowledgments}

This research was supported by the Main Research Program of the Korea Food Research Institute, funded by the Ministry of Science, ICT, \& Future Planning (grant number E0187400).

\section{Supplementary Materials}

Figure S1: cellular viability of MGO-treated SV40MES13 cells. SV40MES13 cells were treated with various concentrations of MGO $(0-0.75 \mathrm{mM})$ for $24 \mathrm{~h}$, and cell viability was measured using the MTT assay. Figure S2: protein levels of autophagy-related genes in MGO-treated SV40MES13 cells. SV40MES13 cells were seeded in a 6-well plate $\left(2 \times 10^{5}\right.$ cells per well). On the next day, the cells were treated with 0 $0.25 \mathrm{mM}$ of MGO for $24 \mathrm{~h}$. The cells were harvested and analyzed by immunoblotting. (Supplementary Materials)

\section{References}

[1] I. Allaman, M. Bélanger, and P. J. Magistretti, "Methylglyoxal, the dark side of glycolysis," Frontiers in Neuroscience, vol. 9, p. 23, 2015.

[2] C. M. Chan, D. Y. Huang, Y. P. Huang et al., "Methylglyoxal induces cell death through endoplasmic reticulum stressassociated ROS production and mitochondrial dysfunction," Journal of Cellular and Molecular Medicine, vol. 20, no. 9, pp. 1749-1760, 2016.

[3] J. Bellier, M.-J. Nokin, E. Lardé et al., "Methylglyoxal, a potent inducer of AGEs, connects between diabetes and cancer," Diabetes Research and Clinical Practice, vol. 148, pp. 200-211, 2019.

[4] H. Wang, J. Liu, and L. Wu, "Methylglyoxal-induced mitochondrial dysfunction in vascular smooth muscle cells," Biochemical Pharmacology, vol. 77, no. 11, pp. 1709-1716, 2009.

[5] A. Moraru, J. Wiederstein, D. Pfaff et al., "Elevated levels of the reactive metabolite methylglyoxal recapitulate progression of type 2 diabetes," Cell Metabolism, vol. 27, no. 4, pp. 926934.e8, 2018.

[6] M. Pastor-Belda, A. J. Fernández-García, N. Campillo et al., "Glyoxal and methylglyoxal as urinary markers of diabetes. Determination using a dispersive liquid-liquid microextraction procedure combined with gas chromatography-mass spectrometry," Journal of Chromatography A, vol. 1509, pp. 43-49, 2017.

[7] N. M. J. Hanssen, J. Westerink, J. L. J. M. Scheijen et al., "Higher plasma methylglyoxal levels are associated with incident cardiovascular disease and mortality in individuals with type 2 diabetes," Diabetes Care, vol. 41, no. 8, pp. 1689-1695, 2018.

[8] K. M. Lee, C. Y. Lee, G. Zhang, A. Lyu, and K. K. M. Yue, "Methylglyoxal activates osteoclasts through JNK pathway leading to osteoporosis," Chemico-Biological Interactions, vol. 308, pp. 147-154, 2019.

[9] Y. A. Shamsaldeen, L. S. Mackenzie, L. A. Lione, and C. D. Benham, "Methylglyoxal, a metabolite increased in diabetes is associated with insulin resistance, vascular dysfunction and neuropathies," Current Drug Metabolism, vol. 17, no. 4, pp. 359-367, 2016.

[10] X. Huang, F. Wang, W. Chen, Y. Chen, N. Wang, and K. von Maltzan, "Possible link between the cognitive dysfunction associated with diabetes mellitus and the neurotoxicity of methylglyoxal," Brain Research, vol. 1469, pp. 82-91, 2012.

[11] D. S. Fosmark, P. A. Torjesen, B. K. Kilhovd et al., "Increased serum levels of the specific advanced glycation end product methylglyoxal-derived hydroimidazolone are associated with retinopathy in patients with type 2 diabetes mellitus," Metabolism, vol. 55, no. 2, pp. 232-236, 2006.

[12] J. M. Forbes and M. E. Cooper, "Mechanisms of diabetic complications," Physiological Reviews, vol. 93, no. 1, pp. 137-188, 2013.

[13] J. Berlanga, D. Cibrian, I. Guillén et al., "Methylglyoxal administration induces diabetes-like microvascular changes and perturbs the healing process of cutaneous wounds," Clinical Science, vol. 109, no. 1, pp. 83-95, 2005. 
[14] P. J. Beisswenger, S. K. Howell, G. B. Russell, M. E. Miller, S. S. Rich, and M. Mauer, "Early progression of diabetic nephropathy correlates with methylglyoxal-derived advanced glycation end products," Diabetes Care, vol. 36, no. 10, pp. 3234-3239, 2013.

[15] J. D. Rabinowitz and E. White, "Autophagy and metabolism," Science, vol. 330, no. 6009, pp. 1344-1348, 2010.

[16] S. Skah, N. Richartz, E. Duthil et al., "cAMP-mediated autophagy inhibits DNA damage-induced death of leukemia cells independent of p53," Oncotarget, vol. 9, no. 54, pp. 3043430449, 2018.

[17] X.-T. Zheng, Z.-H. Wu, Y. Wei et al., "Induction of autophagy by salidroside through the AMPK-mTOR pathway protects vascular endothelial cells from oxidative stress-induced apoptosis," Molecular and Cellular Biochemistry, vol. 425, no. 1-2, pp. 125-138, 2017.

[18] E. N. N'Diaye, K. K. Kajihara, I. Hsieh, H. Morisaki, J. Debnath, and E. J. Brown, "PLIC proteins or ubiquilins regulate autophagy-dependent cell survival during nutrient starvation," EMBO Reports, vol. 10, no. 2, pp. 173-179, 2008.

[19] D. Vizza, A. Perri, G. Toteda et al., "Rapamycin-induced autophagy protects proximal tubular renal cells against proteinuric damage through the transcriptional activation of the nerve growth factor receptor NGFR," Autophagy, vol. 14, no. 6, pp. 1028-1042, 2018.

[20] J. Q. Liu, L. Zhang, J. Yao, S. Yao, and T. Yuan, “AMPK alleviates endoplasmic reticulum stress by inducing the ERchaperone ORP150 via FOXO1 to protect human bronchial cells from apoptosis," Biochemical and Biophysical Research Communications, vol. 497, no. 2, pp. 564-570, 2018.

[21] L. Fang, X. Li, Y. Zhong et al., "Autophagy protects human brain microvascular endothelial cells against methylglyoxalinduced injuries, reproducible in a cerebral ischemic model in diabetic rats," Journal of Neurochemistry, vol. 135, no. 2, pp. 431-440, 2015.

[22] Y. Liu, J. Huang, X. Zheng et al., "Luteolin, a natural flavonoid, inhibits methylglyoxal induced apoptosis via the mTOR/4EBP1 signaling pathway," Scientific Reports, vol. 7, no. 1, p. 7877, 2017.

[23] D. H. Lee, J. Ahn, Y. J. Jang, T. Y. Ha, and C. H. Jung, "Oleic acid-induced defective autolysosome shows impaired lipid degradation," Biochemical and Biophysical Research Communications, vol. 513, no. 3, pp. 553-559, 2019.

[24] J. H. Jang, E. A. Kim, H. J. Park et al., "Methylglyoxal-induced apoptosis is dependent on the suppression of c-FLIP $\mathrm{L}_{\mathrm{L}}$ expression via down-regulation of p65 in endothelial cells," Journal of Cellular and Molecular Medicine, vol. 21, no. 11, pp. 27202731, 2017.

[25] Y.-C. Chang, M.-C. Hsieh, H.-J. Wu, W.-C. Wu, and Y.-H. Kao, "Methylglyoxal, a reactive glucose metabolite, enhances autophagy flux and suppresses proliferation of human retinal pigment epithelial ARPE-19 cells," Toxicology In Vitro, vol. 29, no. 7, pp. 1358-1368, 2015.

[26] A. L. Dafre, A. E. Schmitz, and P. Maher, "Methylglyoxalinduced AMPK activation leads to autophagic degradation of thioredoxin 1 and glyoxalase 2 in HT22 nerve cells," Free Radical Biology \& Medicine, vol. 108, pp. 270-279, 2017.

[27] S. R. Yoshii and N. Mizushima, "Monitoring and measuring autophagy," International Journal of Molecular Sciences, vol. 18, no. 9, p. $1865,2017$.
[28] Y. Liu and B. Levine, "Autosis and autophagic cell death: the dark side of autophagy," Cell Death and Differentiation, vol. 22, no. 3, pp. 367-376, 2015.

[29] P. Codogno and A. J. Meijer, "Atg5: more than an autophagy factor," Nature Cell Biology, vol. 8, no. 10, pp. 1045-1047, 2006.

[30] C. H. Jung, S.-H. Ro, J. Cao, N. M. Otto, and D.-H. Kim, "mTOR regulation of autophagy," FEBS Letters, vol. 584, no. 7, pp. 1287-1295, 2010.

[31] A. M. J. Sanchez, R. B. Candau, and H. Bernardi, "FoxO transcription factors: their roles in the maintenance of skeletal muscle homeostasis," Cellular and Molecular Life Sciences, vol. 71, no. 9, pp. 1657-1671, 2014.

[32] A. M. Sanchez, A. Csibi, A. Raibon et al., "AMPK promotes skeletal muscle autophagy through activation of forkhead FoxO3a and interaction with Ulk1," Journal of Cellular Biochemistry, vol. 113, no. 2, pp. 695-710, 2012.

[33] M. C. Maiuri, E. Zalckvar, A. Kimchi, and G. Kroemer, "Selfeating and self-killing: crosstalk between autophagy and apoptosis," Nature Reviews Molecular Cell Biology, vol. 8, no. 9, pp. 741-752, 2007.

[34] C. C. Thoreen, S. A. Kang, J. W. Chang et al., "An ATPcompetitive mammalian target of rapamycin inhibitor reveals rapamycin-resistant functions of mTORC1," Journal of Biological Chemistry, vol. 284, no. 12, pp. 8023-8032, 2009.

[35] X. Lin, L. Han, J. Weng, K. Wang, and T. Chen, "Rapamycin inhibits proliferation and induces autophagy in human neuroblastoma cells," Bioscience Reports, vol. 38, no. 6, article BSR20181822, 2018.

[36] I. Tamargo-Gómez and G. Mariño, “AMPK: regulation of metabolic dynamics in the context of autophagy," International Journal of Molecular Sciences, vol. 19, no. 12, article $3812,2018$.

[37] D. M. Thomson, S. T. Herway, N. Fillmore et al., "AMPactivated protein kinase phosphorylates transcription factors of the CREB family," Journal of Applied Physiology, vol. 104, no. 2, pp. 429-438, 2008.

[38] L. el-Houjeiri, E. Possik, T. Vijayaraghavan et al., "The transcription factors TFEB and TFE3 link the FLCN-AMPK signaling axis to innate immune response and pathogen resistance," Cell Reports, vol. 26, no. 13, pp. 3613-3628.e6, 2019.

[39] G. Nystrom and C. Lang, "Sepsis and AMPK activation by AICAR differentially regulate foxO- $1,-3$ and -4 mRNA in striated muscle," International Journal of Clinical and Experimental Medicine, vol. 1, no. 1, pp. 50-63, 2008.

[40] M. Saline, L. Badertscher, M. Wolter et al., "AMPK and AKT protein kinases hierarchically phosphorylate the N-terminus of the FOXO1 transcription factor, modulating interactions with 14-3-3 proteins," Journal of Biological Chemistry, vol. 294, no. 35, pp. 13106-13116, 2019.

[41] F. C. Brosius III, C. E. Alpers, E. P. Bottinger et al., "Mouse models of diabetic nephropathy," Journal of the American Society of Nephrology, vol. 20, no. 12, pp. 2503-2512, 2009. 